\title{
Fuzzy Control of a Robotic Arm using EMG Signals
}

\author{
M. Hidalgo*, G. Tene*, and A. Sánchez*Member, IEEE \\ * Departamento de Automatización y Control Industrial, Escuela Politécnica Nacional \\ Ladrón de Guevara E11-253, Quito Ecuador \\ Email: galoftene@mail.com
}

\begin{abstract}
This paper presents the control design of a robotic arm employing Fuzzy algorithms to interpret electromiographic (EMG) signals from the Flexor Carpi Radialis, Extensor Carpi Radialis and Biceps Brachii muscles. The control and aquisition systems is composed of a microprocessor, analog filtering, digital filtering and frequency analysis, and finally a fuzzy control system. The system has been implemented over a MICROCHIP PIC $16 F 876$ and LabVIEW.

Copyright $@ 2005$ IEEE
\end{abstract}

\section{INTRODUCTION}

This paper presents the control design of a robotic arm employing Fuzzy algorithms to interpret electromyographic (EMG) signals from the Flexor Carpi Radialis, Extensor Carpi Radialis and Biceps Brachii muscles. The control and aquisition system is composed of a microprocessor, analog filtering, digital filtering and frequency analysis, and finally a fuzzy control system. The system has been implemented over a MICROCHIP PIC 16F876 and LabVIEW.

The processing of EMG signals has been discussed in several works as from de Luca [1], where the author gives an extensive explanation on how these signals are generated, and how they can be modelled. The difficulty to acquire the EMG signals have led to research in the use optimal estimation for this purpose. Work in this area is extensive, as for example the work of Hogan and Mann [2], [3]. The correlation between myographic signals and motion has been investigated by several researchers as for example the works presented in [4], [5], [6]. How to acquire the signal via surface eletrodes is extensively presented by de Luca in [7], [8].

The use of these signals for several applications have also been reported by several authors. Rodríguez [9] employs a computer simulation to investigate if a proesthetic limb can be adapted to a particular person before this device is acquired or constructed. This software employs pre-processed signals acquired using a device developed by de la Rosa [10]. Zahedi [11] for example employs these signals to simulate the motion control of a full complex hand, while in [12], a similar device is implemented and experimental results are presented.

The work presented in this paper employs EMG signals acquired using surface $\mathrm{Ag} / \mathrm{AgCl}$ disposable electrodes. The signals are then amplified with a $46 \mathrm{~dB}$ instrumentation amplifier implemented using a drivenright-leg circuit to enhance the CMRR. After the amplification phase, the signals are then passed through highpass filter to eliminate bias and a second amplification stage. Prior to the analog to digital conversion the signals are passed through an anti-aliasing filter. The conversion is performed employing a MICROCHIP PIC 16F876 AD interface. Sampling is performed at $10 \mathrm{kHz}$ with a 19.5 $\mathrm{mV}$ resolution and 8 bit quantification.

Once the signals have been acquired, they are transmitted to a computer runnig LabVIEW via RS-232 at $115.2 \mathrm{Kbps}$. The software implemented in LabVIEW performs additional filtering to eliminate low frequencies residual componentes $(60 \mathrm{~Hz})$, and all fuzzy algorithms to generate control signals which are later transmitted back to the microprocessor to drive the robotic arm.

The paper is organized in the following way: the theory behind the EMG signals produced by the muscles is presented in section 2 . Subsequently the signal processing, is presented in section 3 . The fuzzy controllers implementations are presented in section 4 , and finally at the end of the paper, conclusions are drawn.

\section{EleCtromyographic Signals}

This section introduces the nature of the EMG signals and their correlation with motion. The section is based on the work of de Luca [7].

\section{A. Nature of the EMG Signals}

In the human body, the signal required to move a muscle is generated in the spine. This signal is transmitted through neurones. The neurone that is joined to 
microcontroller to drive the servomotors that move the robotic arm.

The software operates with a data block of 2000 samples per channel. After the passing through the stop band filter, the EMG signals are automatically analyzed in the frequency-domain performing a power spectrum decomposition using an FFT algorithm, which delivers the EMG spectral content between DC and $5 \mathrm{kHz}$.

Employing the power spectrum, the less representative frequency components are eliminated, so only the most significant information concerning the muscle contraction is left. Components under $20 \mathrm{~Hz}$ are discarded because they are correlated with the electrodes movements in the skin surface[13]. This noise is commonly called motion artifacts.

According to some biomedical research the most representative information about limb position is contained in the frequency range under $500 \mathrm{~Hz}$ [14]. The recovered frequency content is employed to calculate an average RMS value of the EMG, mainly due to the non-stationary properties of the EMG. Thus, to improve the reliability of the measurement, the mean of a set of ten measurements is employed. Therefore the control system will actually employ an estimation of the expected value of the EMG signal. The equation to calculate the expected value is presented in Equation (1).

$$
\begin{aligned}
V_{R M S} & =E\left\{V_{r m s}\right\} \\
& =\frac{1}{10} \sum_{i}^{10} V_{r m s_{i}}
\end{aligned}
$$

The expected RMS value has a relatively lineal relationship with the analyzed muscle contraction. This lineal relationship was obtained performing several experiments, in which the EMG signal was correlated with the muscle motion and position. To determinate the position of the wrist, the muscles Extensor Carpi Radialis and the Flexor Carpi Radialis were analyzed. Considering that when the Extensor Carpi is contracted, it produces the wrist extension, whereas the contraction of the Flexor Carpi does not produce relevant information. Similarly, when the Flexor Carpi Radialis is contracted, it produces the wrist flexion, and the contraction of Extensor Carpi Radialis does not produce relevant information.

The fuzzy controller is designed using the relationship between the values of the average RMS obtained per muscle (Flexor Carpi Radialis and Extensor Carpi Radialis) and the wrist position obtained via experimentation. Figure (5) shows the EMG signal, its Frequency

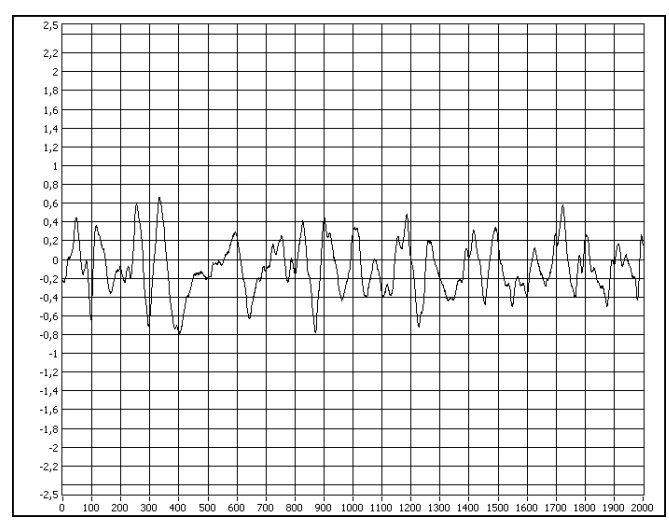

(a) Time Series

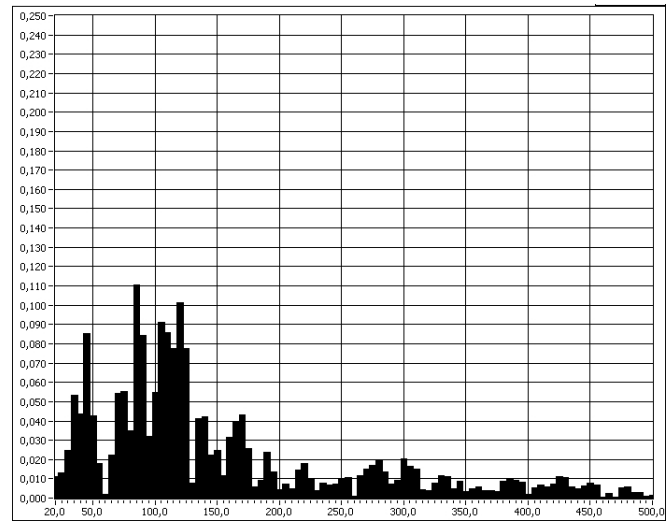

(b) Power Spectrum

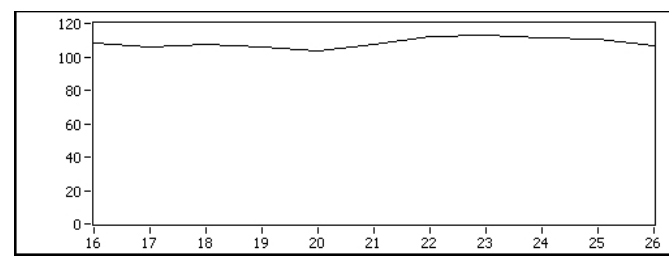

(c) RMS value

Fig. 5. Flexor Carpi Radialis Analysis

content, and the calculated expected RMS values for the Flexor Carpi Radialis muscle.

The information provided by both EMGs are also employed to control the opening and closing of the robotic-arm gripper. The exact position of the gripper cannot be controlled, because this will require the use of the EMG signals from additional muscles which have not been considered in this work. The controller is designed to open the gripper when the hand is totally relaxed and close the gripper when the hand is closed.

To determine the control signal for the elbow position, the only muscle analyzed is the Biceps Brachii. However, experimental and theoretical results [9] indicate that to improve the position estimation and thus the control of 
the elbow of the robotic arm, not only the expected RMS value is necessary but also the variance.

The variance calculation significantly improves the estimation of a change in position (motion) of the muscle. If the variance has a low value $(<150)$, the fuzzy controller provides a stable position for the robotic arm. Thus the robotic arm will not follow the motion of the muscle in each instant, but when the muscle has reached a final position. The position calculated by the fuzzy controller is then transmitted to the microcontroller to drive the arm to the required position.

\section{FuZZY CONTROLlers}

The controller is composed of two fuzzy control systems for the movement of the wrist and the elbow, and an ON/OFF controller for the gripper.

The fuzzy controller for the wrist receives the RMS expected values of the Flexor Carpi Radialis and the Extensor Carpi Radialis. This controller is configured in the following way: (i) singleton fuzzyfication, (ii) 5 triangular membership functions, (iii) COG defuzzification, and (iv) Mamdami inference engine usign the Max$\operatorname{Min}\{\}$ operators. The controller output is the calculated position. The Fuzzy Associative Memory for this controller is presented in Table (I). In this table the following convention of symbols have been used: (i) L for Low (ii) LL for Very Low (iii) $\mathrm{H}$ for High (iv) HH Very High (v) $\mathrm{M}$ for Medium (vi) I for Left (vii) D for Right (viii) C for Centre.

For the elbow the input is the expected rms value and the variance of the Biceps Brachii. The controller is configured in a similar way as for the wrist. The Fuzzy Associative Memory for this controller is presented in Table (II). In this table the following convention of symbols have been used: (i) B for Low (ii) BB for Very Low (iii) A for High (iv) AA Very High (v) M for Medium.

Figure (6) shows a picture of the robotic arm employed, and Figure (7) shows the complete system.

\section{CONClusions And Further Work}

The analysis of EMG signals applied to robotics control are a field of quick development in the last years. The treatment of these signals is complex. This work has presented an application of the use of EMG signals for the control of a robotic arm, employing fuzzy algorithms. Even though the system works in an acceptable way, there is however room for several improvements. The robotic arm moves accordingly to the movements of the three muscles, however, the processing time is quite
TABLE I

FuZZY Associative MEMORY FOR WRIST CONTROL

\begin{tabular}{|c|c|c|c|}
\hline No. & FCR & ECR & Inference \\
\hline 1 & PLL & PLL & $\mathrm{PC}$ \\
\hline 2 & PLL & PL & $\mathrm{PC}$ \\
\hline 3 & PLL & PM & PD \\
\hline 4 & PLL & $\mathrm{PH}$ & PDD \\
\hline 5 & PLL & PHH & PDDD \\
\hline 6 & PL & PLL & PI \\
\hline 7 & PL & PL & NONE \\
\hline 8 & PL & PM & PD \\
\hline 9 & PL & PH & PDDD \\
\hline 10 & PL & PHH & PDDD \\
\hline 11 & PM & PLL & PII \\
\hline 12 & PM & PL & PII \\
\hline 13 & PM & PM & PC \\
\hline 14 & PM & PH & PDD \\
\hline 15 & PM & PHH & PDDD \\
\hline 16 & $\mathrm{PH}$ & PLL & PII \\
\hline 17 & $\mathrm{PH}$ & PL & PIII \\
\hline 18 & $\mathrm{PH}$ & PM & PIII \\
\hline 19 & $\mathrm{PH}$ & $\mathrm{PH}$ & NONE \\
\hline 20 & $\mathrm{PH}$ & PHH & NONE \\
\hline 21 & PHH & PLL & PIII \\
\hline 22 & PHH & PL & PIII \\
\hline 23 & PHH & PM & PIII \\
\hline 24 & PHH & $\mathrm{PH}$ & PIII \\
\hline 25 & PHH & PHH & NONE \\
\hline
\end{tabular}

TABLE II

Fuzzy Associative Memory FOR ELBow CONTROL

\begin{tabular}{cccc}
\hline No. & RMS & Variance & Inference \\
\hline 1 & BB & B & PBB \\
2 & BB & M & PBB \\
3 & BB & A & PBB \\
4 & B & B & PB \\
5 & B & M & PB \\
6 & B & A & PB \\
7 & M & B & PM \\
8 & M & M & PM \\
9 & M & A & PM \\
10 & A & B & PA \\
11 & A & M & PA \\
12 & A & A & PA \\
13 & AA & B & PAA \\
14 & AA & M & PAA \\
15 & AA & A & PAA \\
\hline
\end{tabular}


